\title{
THE MEIJER TRANSFORMATION OF GENERALIZED FUNCTIONS
}

\author{
E.L. KOH \\ Department of Mathematics and Statistics \\ University of Regina \\ Regina, Canada S4S OA2 \\ E.Y. DEEBA \\ Department of Applied Mathematical Sciences \\ University of Houston-Downtown \\ Houston, Texas 77002 \\ and
}

M.A. ALI

$\# 1598$, Way 510

Muharraq 205, Bahrain

(Received June 2, 1986)

ABSTRACT. This paper extends the Meijer transformation, $M_{\mu}$, given by $\left(M_{\mu} f\right)(p)=\frac{2 p}{\Gamma(1+\mu)} \int_{0}^{\infty} f(t)(p t)^{\mu / 2} K_{\mu}(2 \sqrt{p t}) d t$,

where $f$ belongs to an appropriate function space, $\mu \varepsilon(-1, \infty)$ and $k_{\mu}$ is the modified Bessel function of third kind of order $\mu$, to certain generalized functions. A testing space is constructed so as to contain the kernel, $(p t)^{\mu / 2} K_{\mu}(2 \sqrt{p t})$, of the transformation. Some properties of the function space and its dual are derived. The generalized Meijer transform, $\bar{M}_{\mu} f$, is now defined on the dual space. This transform is shown to be analytic and an inversion theorem, in the distributional sense, is established.

KEY WOROS AND PHRASES. Meijer transform, generalized functions, Bessel differential operator, Schwartz distributions, Operational calculus.

1980 MATHEMATICS SUBJECT CLASSIFICATION CODES. 46F12, 44A15, 46F05, 33A40. 
1. Iiv'RRODUCTION. In order to develop an operational calculus for the Bessel differential operator $B_{\mu}=t^{-\mu} D t^{1+\mu} D$, where $\mu \varepsilon(-1, \infty)$ and $D=\frac{d}{d t}$, Conlan and Koh [1] used the Meijer transformation $M_{\mu} f$ given above. The operational formulas obtained mirrored those obtained by Koh [2] by means of a Mikusinski-type calculus. The latter is algebraic in approach and allows for certain convolution operators not covered by the classical integral transform $M_{\mu} f$. To remedy this situation, the Meijer transformation has to be extended to generalized functions. This is the object of the present work.

The idea is to construct a testing function space $M_{\mu, \gamma}$ which contains the kernel (pt) ${ }^{\mu / 2} \mathrm{~K}_{\mu}(2 \sqrt{\mathrm{pt}})$. The generalized Meijer transformation $\bar{M}_{\mu} f$ is now defined on the dual space . ${ }^{\prime} \mu, \gamma$ as follows: for $\mathrm{f}_{\mathcal{M}} \mathrm{M}^{\prime}{ }_{\mu, \gamma}$

$$
\left(\bar{M}_{\mu} f\right)(p)=\frac{2 p}{\Gamma(1-\mu)}<f(t),(p t)^{\mu / 2} K_{\mu}(2 \sqrt{p t})>,
$$

where $\mu \varepsilon(-\infty, 1)$. The definition in (1.1) coincides

with the classical transformation whenever $f$ is a regular distribution, that is, one that can be represented by a suitable integrable function. We note that there are various extensions of classical transforms in which the operational calculus is geared for other Bessel-type operators among which are the K-transform [3] and the Hankel transform [3], [4], [5] and [6].

In section two we shall describe the testing function space $M_{\mu, \gamma^{\prime}}$ its dual and study some of their properties. While in section three we will give the definition of the generalized Meijer transform, show its analyticity in some region of the complex plane, and then derive an inversion theorem.

Throughout the sequel we shall make use of the following notations and facts and are stated for the sake of completeness. 
We shall denote the interval $(0, \infty)$ by $I$, the Bessel differential operator $t^{\mu} D t^{1-\mu} D$ by $B_{-\mu}$ and its $k$-iterate by $B_{-\mu}^{k}$. It follows that

$B_{-\mu}^{k} \phi(t)=\left[t^{k} D_{t}^{2 k}+c_{1} t^{k-1} D_{t}^{2 k-1}+c_{2} t^{k-2} D_{t}^{2 k-2}+\ldots+c_{k} D_{t}^{k}\right] \phi(t)$,

where the c's are suitable functions of $\mu$ only.

The modified Bessel functions $I_{\mu}(2 \sqrt{p t})$ and $k_{\mu}(2 \sqrt{p t})$ of first and third kinds are defined by (see watson [7])

$$
I_{\mu}(2 \sqrt{p t})=\sum_{k=0}^{\infty} \frac{(p t)^{k+\mu / 2}}{k ! \Gamma(k+1+\mu)}, \mu \text { any real number, }
$$

and

$$
\begin{aligned}
& k_{\mu}(2 \sqrt{p t})=\left\{\begin{array}{l}
\frac{\pi}{2 \sin \mu \pi}\left(\sum_{k=0}^{\infty} \frac{(p t)^{k-\mu / 2}}{k ! \Gamma(k+1-\mu)}-\sum_{k=0}^{\infty} \frac{(p t)^{k+\mu / 2}}{k ! \Gamma(k+1+\mu)}\right), \mu \text { not an integer, } \\
-\mu-1 \\
\frac{1}{2} \sum_{k=0} \frac{(-1)^{k}(-\mu-k-1) !}{k !}(p t)^{k+\mu / 2}+(-1)^{-\mu} \sum_{k=0}^{\infty} \frac{(p t)^{k-\mu / 2}}{k !(-\mu+k) !} \cdot
\end{array}\right. \\
& {\left[-\log c(\sqrt{p t})+\frac{1}{2}\left(\sum_{i=1}^{k} \frac{1}{i}+\sum_{i=1}^{-\mu+k} \frac{1}{i}\right)\right], \mu \text { integer }}
\end{aligned}
$$

$c=e^{\gamma}(\gamma$ is Euler's constant) and the finite sums are taken to be equal to zero whenever the upper limit is less than the lower limit.

Further,

$$
\begin{aligned}
& B_{-\mu}^{k}\left((p t)^{\mu / 2} K_{\mu}(2 \sqrt{p t})\right)=p^{k}(p t)^{\mu / 2} K_{\mu}(2 \sqrt{p t}), \\
& B_{-\mu}^{k}\left((p t)^{\mu / 2} I_{\mu}(2 \sqrt{p t})\right)=p^{k}(p t)^{\mu / 2} I_{\mu}(2 \sqrt{p t}) .
\end{aligned}
$$

Finally, the following asymptotic expansions of $\mathrm{K}_{\mathrm{U}}(2 \sqrt{\mathrm{pt}})$ and $I_{\mu}(2 \sqrt{p t})$ will be used repeatedly (see watson [7]):

$$
K_{j}(2 \sqrt{p t})=\frac{\sqrt{\pi}}{2}(p t)^{-1 / 4} e^{-2 \sqrt{p t}}\left[1+0\left(\mid p t i^{-1 / 2}\right)\right],-\pi<\operatorname{argp}-\pi \quad(1.7)
$$

and 


$$
I_{\mu}(2 \sqrt{p t})=\left\{\begin{array}{c}
\frac{1}{2 \sqrt{\pi}}(p t)^{-1 / 4}\left(e^{2 \sqrt{p t}}+i e^{-2 \sqrt{p t}+i \mu \pi}\right)\left[1+0\left(|p t|^{-1 / 2}\right)\right] \\
-\pi / 2<\operatorname{argp}<\frac{3 \pi}{2} \\
\frac{1}{2 \sqrt{\pi}}(p t)^{-1 / 4}\left(e^{2 \sqrt{p t}}-i e^{-2 \sqrt{p t}-i \mu \pi}\right)\left[1+0\left(|p t|^{-1 / 2}\right)\right] \\
-3 \pi / 2<\operatorname{argp}<\pi / 2 .
\end{array}\right.
$$

2. The Testing Function Space $M_{\mu, \gamma}$ and Its Dual. In this section we shall define the testing functions space $M_{\mu, \gamma}$, its dual and study some of their properties.

Let $\gamma$ be any real number and $\mu \varepsilon(-\infty, 1)$. Define

$$
M_{\mu, \gamma}=\left\{\phi \varepsilon C^{\infty}(I) \mid \lambda_{\gamma, k}^{\mu}(\phi)<\infty\right\}
$$

where

$$
\lambda_{\gamma, k}^{\mu}(\phi)=\sup _{0<t<\infty}\left|e^{\gamma \sqrt{t}} t^{1-\mu_{B} k} \phi(t)\right|, k=0,1,2, \ldots .
$$

In view of the weight function $e^{\gamma \sqrt{t}} t^{1-\mu}$ one may think of the elements of $M_{\mu, \gamma}$ as those smooth functions on $I$ that grow no faster than the exponential function $e^{\gamma \sqrt{t}}$ for large $t$ and behave like power functions near the origin. As will be seen in Lemma 2.1 below, the kernel of the Meijer transform is of this type.

The family $\left\{\lambda_{\gamma, k}^{\mu} \mid k=0,1,2, \ldots\right\}$ is a countable multinorm. Indeed, $\lambda_{\gamma, k}^{\mu}$ is a semi-norm for each $k$ and $\lambda_{\gamma, 0}^{\mu}$ is a norm on $M_{\mu, \gamma^{*}}$ We assign to $M_{\mu, \gamma}$ the topology generated by this family of multinorms. Thus $M_{\mu, \gamma}$ is a countably normed space (a fundamental space and members of the dual space $M^{\prime}{ }_{\mu, \gamma}$ are generalized functions). A sequence $\left\{\phi_{\nu}\right\}$ is cauchy in $M_{\mu, \gamma}$ if $\phi_{\nu} \varepsilon M_{\mu, \gamma}$ for all $\nu$ and for every $k=0,1,2, \ldots, \lambda_{\gamma, k}^{\mu}\left(\phi_{\nu}-\phi_{\xi}\right)+0$ as $\nu, \xi \rightarrow \infty$ independently. We shall now show that the kernel, $(p t){ }^{\mu / 2} K_{\mu}(2 \sqrt{p t})$, is a member of $M_{\mu, \gamma^{*}}$

Lemma 2.1. For any fixed complex number $p$ such that $p \neq 0$, $-\pi<\arg \mathrm{p}<\pi$ and $\operatorname{Re} 2 \sqrt{\mathrm{p}}>\gamma_{,}(\mathrm{pt})^{\mu / 2} \mathrm{~K}_{\mu}(2 \sqrt{\mathrm{pt}}) \varepsilon M_{\mu, \gamma}$ Proof. Under the hypothesis on $p, k_{\mu}(2 \sqrt{p t})$ is an analytic 
function of $t$ on the right half plane and hence a smooth function on $I$. It remains to show that $\lambda_{\gamma, k}^{\mu}\left((p t)^{\mu / 2} K_{\mu}(2 \sqrt{p} \bar{E})\right)<\infty$ for $k=0,1,2, \ldots$. From $(1.5)$, we have

$\lambda_{\gamma, k}^{\mu}\left((p t)^{\mu / 2} K_{\mu}(2 \sqrt{p t})\right)=\sup _{t \in I}\left|e^{\gamma \sqrt{t}} t^{1-\mu} p^{k}(p t)^{\mu / 2} K_{\mu}(2 \sqrt{p t})\right|$.

We will consider two cases: for small $t$ and for large $t$. For $t$ small and $\mu$ not equal to an integer the asymptotic expansion of $\mathrm{K}_{\mu}(2 \sqrt{\mathrm{pt}})$ in $(1.4)$ yields

$$
\begin{aligned}
& \left|e^{\gamma \sqrt{t}} t^{1-\mu} p^{k}(p t)^{\mu / 2} k_{\mu}(2 \sqrt{p t})\right| \leq A_{\mu} e^{\gamma / \sqrt{t}}|p|^{k+\mu-1}\left[|p t|^{1-\mu}+|p t|^{2-\mu}+\ldots\right]+ \\
& \quad+c_{\mu} e^{\gamma \sqrt{t}}|p|^{k+\mu-1}\left[|p t|+|p t|^{2}+|p t|^{3}+\ldots\right]
\end{aligned}
$$

where $A_{\mu}$ and $C_{\mu}$ are functions of $\mu$ only. Since $\mu<1$, there exists a real number $T<1$ such that for $|\mathrm{pt}|<\mathrm{T}$, the right hand side of (2) is bounded by a constant. For $t$ small for for $\mu=0,-1,-2, \ldots$ the expansion in (1.4) yields

$$
\begin{aligned}
& \left|e^{\gamma \sqrt{t}}{ }^{1-\mu} p^{k}(p t) \mu / 2 K_{\mu}(2 \sqrt{p t})\right| \leq A_{\mu}^{\prime} e^{\gamma \sqrt{t}}|p|^{k+\mu-1}\left[|p t|+|p t|^{2}+\ldots+|p t|^{-\mu}\right]+ \\
& \quad+c_{\mu}^{\prime} e^{\gamma \sqrt{t}}|p|^{k+\mu-1}|\log c(p t)|\left[|p t|^{1-\mu}+|p t|^{2-\mu}+|p t|^{3-\mu}+\ldots\right] \\
& \quad+E_{\mu} e^{\gamma \sqrt{t}}|p|^{k+\mu-1}\left[|p t|^{1-\mu}+|p t|^{2-\mu}+|p t|^{3-\mu}+\ldots\right]
\end{aligned}
$$

where, $A_{\mu}^{\prime}, C_{\mu}^{\prime}$ and $E_{\mu}$ are constants depending upon $\mu$ only. Applying L'Hopital's rule to the second term in (2.3) and using the fact that $\mu<1$, we imply that there is a real number $T<1$ such that for $|p t|<T$, the right hand side of (2.3) is again bounded by a constant. Thus the left side of (2.2) is bounded for small $t$ and $\mu<1$ for all $k=0,1,2, \ldots$

We now consider the finiteness of (2.1) for large $t$. By employing (1.7), we obtain, for $-\pi<\arg \mathrm{p}<\pi$ and for $|\mathrm{pt}|>\mathrm{T}$,

$$
\begin{gathered}
\left|e^{\gamma \sqrt{t}} t^{1-\mu_{p} k}(p t)^{\mu / 2}{ }_{\mu}(2 \sqrt{p t})\right| \leq E_{\mu}^{\prime}|p|^{k+\mu-1} e^{(\gamma-\operatorname{Re} 2 \sqrt{p}) \sqrt{t}}|p t|^{3 / 4-\mu / 2} \\
{\left[1+0\left(|p t|^{-1 / 2}\right)\right] .}
\end{gathered}
$$


where $E_{\mu}^{\prime}$ is a constant depending upon $\mu$ only. Since $\mu<1$ and $\gamma<\operatorname{Re} 2 \sqrt{\mathrm{p}}$, the right hand side of the last expression is bounded by a constant for $|\mathrm{pt}|>\mathrm{T}$.

Thus in either case we have shown that $\lambda_{\gamma, k}^{\mu}\left((p t)^{\mu / 2} k_{\mu}(2 \sqrt{p t})\right)<$ for all $k=0,1,2, \ldots$. Therefore, $(p t)^{\mu / 2_{K}}{ }_{\mu}(2 \sqrt{p t}) \varepsilon M_{\mu, \gamma}$. By employing the following fact

$D_{t}\left[(p t) \pm \mu / 2 K_{\mu}(2 \sqrt{p t})\right]=-p(p t) \pm \mu / 2-1 / 2 K_{\mu I}(2 \sqrt{p t})$

and by arguments similar to the proof of Lemma 2.1, we have

Lemma 2.2. For $p \neq 0,-\pi<\arg p<\pi$ and $\operatorname{Re} 2 \sqrt{p}>\gamma$, $\mathrm{D}_{t}^{\mathrm{k}}\left[(\mathrm{pt})^{\mu / 2} \mathrm{~K}_{\mu}(2 \sqrt{\mathrm{pt}})\right] \varepsilon M_{\mu, \gamma^{*}}$. Further, by symmetry, it follows that $D_{p}^{k}\left[(p t)^{\mu / 2} K_{\mu}(2 \sqrt{p t})\right] \varepsilon M_{\mu, \gamma^{*}} \quad\left(D^{k}\right.$ is the k-iterate of $\left.D\right)$.

In the next few lemmas we shall investigate some properties of the space $M_{\mu, \gamma}$ and its dual.

Lemma 2.3. The space $M_{\mu, \gamma}$ is complete.

Proof. Let $\left\{\phi_{\nu}\right\}$ be a Cauchy sequence in $M_{\mu, \gamma^{*}}$ Then $\lambda_{\gamma, k}^{\mu}\left(\phi_{\nu}\right)<\infty$ for all $k=0,1,2, \ldots$. Hence on every compact subset $K$ of $I, B_{-\mu}^{k}\left(\phi_{\nu}\right)$ converges uniformly for each $k=0,1,2, \ldots$. Let $\phi(t)$ be a smooth function on $I$ and $s$ a fixed point of $I$. Define $\left(D^{-1} \phi\right)(t)=\int_{S}^{t} \phi(\tau) d \tau$. Then $D^{-1} D_{t} \phi(t)=\phi(t)-\phi(s)$. It can be verified that

$$
t^{-1+\mu_{D}-1} t^{-\mu_{B}} \phi_{v}(t)=D_{t} \phi_{v}(t)-\left(\frac{s}{t}\right)^{1-\mu_{D}} \phi_{v}(s)
$$

and

$$
D^{-1} t^{-1+\mu_{D}}-1_{t}-\mu_{B} \phi_{\nu}(t)=\phi_{v}(t)-\phi_{v}(s)-I_{s}(t) D_{s} \phi_{v}(s),
$$

where

$$
L_{s}(t)=\left\{\begin{array}{l}
\frac{1}{\mu} s^{1-\mu}\left(t^{\mu}-s^{\mu}\right), \quad \mu \neq 0 \\
s(\ln t-\ln s), \quad \mu=0 .
\end{array}\right.
$$

Since multiplication by a power of $t$ or the application of $D^{-1}$ preserves uniform convergence on $K$, it follows from (2.5) that $D_{S} \phi_{\nu}$ converges on $K$. From $(2.4)$, it follows that 
$D_{t} \phi_{v}$ converges uniformly on $K$ as $v \rightarrow \infty$. Now the convergence of $D_{t} \phi_{\nu}$ and $B_{-\mu} \phi_{\nu}$ imply the uniform convergence on each $K$ of $\mathrm{D}_{t}^{2} \phi_{\nu}$. Repeating the same argument with $\phi_{\nu}$ and $B_{-\mu} \phi_{\nu}$ replaced by $B_{-\mu}^{k} \phi_{\nu}$ and $B_{-\mu}^{k+1} \phi_{\nu}$ respectively, we deduce that, for each $k=0,1,2, \ldots, D_{t}^{k} \phi_{v}$ converges uniformly on $k$ as $v \rightarrow \infty$. Therefore there exists a smooth function $\phi$ on $I$ such that for each $k=0,1,2, \ldots$ and each $t \varepsilon I, D_{t}^{k} \phi_{\nu}(t)$ converges to $D_{t}^{k} \phi(t)$ as $v \rightarrow \infty$. We will be done if we can show that $\phi(t) \varepsilon M_{\mu, \gamma}$. Since $\left\{\phi_{v}\right\}$ is a Cauchy sequence, it follows that for each $k$ and each $\varepsilon>0$ there is $\mathrm{N}_{\mathrm{k}}$ such that if $\nu, \xi>\mathrm{N}_{\mathrm{k}^{\prime}}$ $\lambda_{\gamma, k}^{\mu}\left(\phi_{\nu}-\phi_{\xi}\right)<\varepsilon / 2$. Further, since $\left|e^{\gamma \sqrt{t}} t_{t}^{1-\mu_{B} k}\left(\phi_{\nu}-\phi\right)\right|+0$ as $\nu \rightarrow \infty$, we have for each $t \varepsilon I$ and $\xi>N_{k}$ that $\left|e^{\gamma \sqrt{t}} t^{1-\mu_{B} k}\left(\phi_{\xi}-\phi\right)\right|<\varepsilon$. Thus $\lambda_{\gamma, k}^{\mu}\left(\phi_{\xi}-\phi\right) \rightarrow 0$ as $\xi \rightarrow \infty$. Since $\lambda_{\gamma, k}^{\mu}(\phi) \leq \lambda_{\gamma, k}^{\mu}\left(\phi_{\xi}-\phi\right)+\lambda_{\gamma, k}^{\mu}\left(\phi_{\xi}\right)$, it follows that $\phi \varepsilon M_{\mu, \gamma}$. Hence $M_{\mu, \gamma}$ is a complete space. (Indeed $M_{\mu, \gamma}$ is a Fréchet space).

Now if $\gamma$ and $\alpha$ are any real numbers with $\gamma<\alpha$, then it is clear that $M_{\mu, \alpha}$ is a subspace of $M_{\mu, \gamma^{-}}$. Further, the restriction of $f \varepsilon M_{\mu, \gamma}$ to $M_{\mu, \alpha}$ belongs to $M_{\mu, \alpha}^{\prime}$. This implies that there is a real number $\sigma_{f}$ such that the restriction of $f$ to $\mu_{\mu, \gamma}$ is in $\mu_{\mu, \gamma}^{\prime}$ if $\gamma>\sigma_{f}$ and is not in $\mu_{\mu, \gamma}^{\prime}$ if $\gamma<\sigma_{f}$.

The real number $\sigma_{f}$ is called the abscissa of definition of $\mathrm{f}$.

For any real numbers $\gamma$ and $\alpha$ with $\gamma<\alpha$, we know that $M_{\mu, \alpha}$ is a subspace of $M_{\mu, \gamma}$. However,

Lemma 2.4. If $\gamma<\alpha$, then $M_{\mu, \alpha}$ is not a dense subspace of $M_{\mu, \gamma}$

Proof. Let $\phi(t)$ be a smooth function given by

$$
\phi(t)= \begin{cases}0, & 0<t<1, \\ e^{-\gamma \sqrt{t}} t_{t}-1+\mu, & 2<t<\infty .\end{cases}
$$

We claim that $\phi(t) \varepsilon M_{\mu, \gamma^{*}}$ Indeed,

$$
\lambda_{\gamma, k}^{\mu}(\phi) \leq \sup _{1<t \leq 2}\left|e^{\gamma \sqrt{t}} t^{1-\mu_{B} k}{ }_{-\mu} \phi(t)\right|+\sup _{2<t<\infty}\left|e^{\gamma \sqrt{t}} t^{1-\mu_{B} k} \phi(t)\right| .
$$


Since $\phi(t)$ is a smooth function, it follows that the first term on the right hand side of $(2.6)$ is bounded. Moreover, from (1.2) and the observation that $D_{t}^{k}\left(e^{-\gamma \sqrt{t}} t^{-1+\mu}\right)=\left[p_{k}\left(t^{-1}\right)+q_{k-1}\left(t^{-1}\right) t^{-1 / 2}\right] t^{-1+\mu} e^{-\gamma \sqrt{t}}$ where $p_{k}\left(t^{-1}\right)$ and $q_{k-1}\left(t^{-1}\right)$ are polynomials in $t^{-1}$. Thus, the second term on the right hand side of (2.6) is bounded. Hence $\lambda_{\gamma, k}^{\mu}(\phi)<\infty$ and $\phi \varepsilon M_{\mu, \gamma}$. Let $N$ a neighborhood of $\phi$ be given by

$$
\mathbf{N}=\mathrm{N}_{0,1 / 2}(\phi)=\left\{\psi \varepsilon M_{\mu, \gamma} \mid \lambda_{\gamma, 0}^{\mu}(\psi-\phi)<1 / 2\right\} .
$$

Now for large $t, \lambda_{\gamma, 0}^{\mu}(\psi-\phi)<1 / 2$ implies that $|\psi(t)|>\frac{1}{2} e^{-\gamma \sqrt{t}} t^{-1+\mu}$. Thus $\left|e^{\alpha \sqrt{t}} t^{1-\mu} \psi(t)\right|=e^{\alpha \sqrt{t}} t^{1-\mu}|\psi(t)|>\frac{1}{2} e^{(\alpha-\gamma) \sqrt{t}}$ which implies that $\lambda_{\alpha, 0}^{\mu}(\psi)$ remains unbounded as $t \rightarrow \infty$. Hence no element of $N$ belongs to $M_{\mu, \alpha^{*}}$ Therefore $M_{\mu, \alpha}$ is not dense in $M_{\mu, \gamma^{*}}$.

Finally, we shall discuss the adjoint of the operator $\mathrm{B}_{-\mu}^{\mathrm{k}}$. It is rather clear that $\mathrm{B}_{-\mu}^{\mathrm{k}}$ is a continuous linear operator on $M_{\mu, \gamma^{*}}$ Let $f \in M_{\mu, \gamma}$ and $\phi \varepsilon M_{\mu, \gamma}$. We define the adjoint $B_{-\mu}^{*}$ of $B_{-\mu}^{k}$ by the equation

$$
\left\langle B_{-\mu}^{*} k, \phi\right\rangle=\left\langle f, B_{-\mu}^{k} \phi\right\rangle k=0,1,2, \ldots .
$$

The continuity and linearity of $B_{-\mu}^{k}$ will imply that $B_{-\mu}{ }^{k} f \varepsilon M_{\mu, \gamma}$ and $B_{-\mu}^{{ }^{*}}$ is a continuous and a linear operator on $\mu_{\mu, \gamma}{ }^{-}$. Further in lieu of equation (1.2), the expression for $B_{-\mu}{ }^{*}$ is given by

$$
B_{-\mu}^{k_{k}}=C_{k} D_{t}^{k}+C_{k-1} t D_{t}^{k+1}+\ldots+C_{1} t^{k-1} D_{t}^{2 k-1}+t^{k} D_{t}^{2 k} \text {, }
$$

where the C's depend only on $\mu$. Moreover, we can show that the adjoint operator $B_{-\mu}^{*}$ is $B_{\mu}^{k}$. Indeed if $k=1$, then (1.2) yields $B_{-\mu}=t D_{t}^{2}+(1-\mu) D_{t}$ while $\left.B_{-\mu}^{*}=t D_{t}^{2}+(1+\mu) D t\right)$. Thus $B_{-\mu}^{*}=B_{\mu}$. Inductively, we obtain $B_{-\mu}^{*}=B_{\mu}^{k}$.

We end this section by the following remarks.

Remark 2.1. If $f(t)$ is locally integrable on $I$ and $f(t) e^{-\gamma \sqrt{t}} t^{-1+\mu}$ is absolutely integrable on $I$, then $f(t)$ generates a regular member $f$ of $M_{\mu, \gamma}$ via 
$\langle f, \phi\rangle=\int_{0}^{\infty} f(t) \phi(t) d t, \quad \phi \varepsilon M_{\mu, \gamma^{*}}$

Remark 2.2. The space $D(I)$, of all smooth complex valued functions on I whose support is contained in a compact subset $\mathrm{K}$ of I equipped with the seminorms

$$
\rho_{n}(\phi)=\sup _{t \in I}\left|D_{t}^{n} \phi(t)\right|
$$

where $\phi \varepsilon D(I)$ is such that supp $\phi \subseteq K$ is a subspace of $M_{\mu, \gamma}$. Further, the restriction of any $f \varepsilon M_{\mu, \gamma}$ to $D(I)$ belongs to $D^{\prime}(I)$.

3. The Generalized Meijer Transform. In this section we shall give the extension of the Meijer transform $M_{\mu} f$ to generalized functions belonging to $\mu_{\mu, \gamma^{*}}$. We shall also prove the analyticity of the transform and exhibit an inversion theorem which is a generalization of the classical inversion formula (see [1]). For $f \varepsilon M_{\mu, \gamma^{\prime}}^{\prime} \mu \varepsilon(-\infty, 1)$ and $p \varepsilon \Omega_{f}=\left\{P \varepsilon \mathbb{C} \mid \operatorname{Re} 2 \sqrt{p}>\gamma>\sigma_{f}, p \neq 0\right.$ $\mid$ arg $\mathrm{p} \mid<\pi\}$, we define the Meijer transform of generalized function by

$$
\left(\bar{M}_{\mu} f\right)(p)=\frac{2 p}{\Gamma(1-\mu)}<f(t),(p t)^{\mu / 2} K_{\mu}(2 \sqrt{p t})>
$$

In (3.1) if $f(t)$ is a regular generalized function (see Remark 2.1) then we obtain the classical Meijer transform

$$
\left(M_{\mu} f\right)(p)=\frac{2 p}{\Gamma(1-\mu)} \int_{0}^{\infty} f(t)(p t)^{\mu / 2} K_{\mu}(2 \sqrt{p t}) d t
$$

where $i \varepsilon(-1, \infty)$.

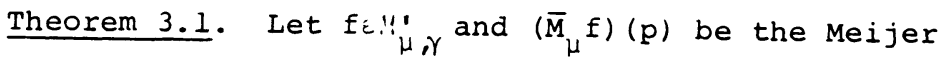
transform in (3.1) for $p \varepsilon \Omega_{f}$. Then

$$
\bar{M}_{\mu}\left(B_{\mu}^{k} f\right)(p)=p^{k} \bar{M}_{\mu}(f)(p) \text {. }
$$

Proof. For $p \varepsilon \Omega_{f}, k=0,1,2, \ldots$, we have

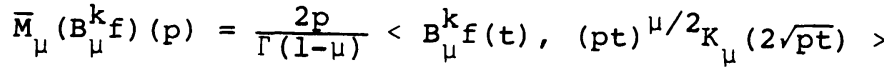




$$
\begin{aligned}
& =\frac{2 p}{\Gamma(1-\mu)}<f(t), B_{-\mu}^{k}(p t)^{\mu / 2} K_{\mu}(2 \sqrt{p t})> \\
& =\frac{2 p}{\Gamma(1-\mu)}<f(t), p^{k}(p t)^{\mu / 2} K_{\mu}(2 \sqrt{p t})> \\
& =p^{k} \bar{M}_{\mu}(f)(p)
\end{aligned}
$$

by equations (1.5) and (3.1) respectively.

Remark 3.1. Theorem 3.1 is the basis for an operational calculus for the operator $B_{\mu}$. It is the generalized function analogue of the differentiation theorem of Conlan and Koh ([1] Theorem 2, page 149).

We shall next prove the analyticity of the transform $\bar{M}_{\mu}(f)$. However, we need the follow lemma.

Lemma 3.1. Let $\gamma$ be any real number and $p$ be a fixed point in $\Omega_{f}$. Then

$$
\sup _{0<t<\infty}\left|e^{\gamma \sqrt{t}}(p t)^{1-\mu / 2} K_{\mu}(2 \sqrt{p t})\right| \leq c_{\mu}\left(1+|p|^{-\mu / 2+3 / 4}\right) .
$$

where $C_{\mu}$ is a constant depending only on $\mu$.

Proof. The idea of the proof is similar to that of

Lemma 2.1. Since $K_{\mu}(2 \sqrt{\mathrm{pt}})$ is analytic except at $t=0$ and $t=\infty$, we need only to show the boundedness of $e^{\gamma \sqrt{t}}(p t)^{1-\mu / 2} K_{\mu}(2 \sqrt{p t})$ as $t \rightarrow 0$ and as $t \rightarrow \infty$. Employing the series expansion of $K_{\mu}(2 \sqrt{p t})(1.4)$ with $\mu \neq 0,-1,-2, \ldots$, we obtain

$$
\begin{aligned}
\left|e^{\gamma \sqrt{t}}(p t)^{1-\mu / 2} k_{\mu}(2 \sqrt{p t})\right| & \leq A_{\mu} e^{\gamma \sqrt{t}}\left[|p t|^{1-\mu}+|p t|^{2-\mu}+|p t|^{3-\mu}+\ldots\right] \\
& +A_{\mu}^{\prime} e^{\gamma \sqrt{t}}\left[|p t|+|p t|^{2}+|p t|^{3}+\ldots\right]
\end{aligned}
$$

where $A_{\mu}$ and $A_{\mu}^{\prime}$ are constants depending upon $\mu$ only. For $T<1$ such that $|p t|<T$, the right hand side of the last expression is bounded by a constant $R_{\mu}$ independent of $p$ and $t$. Similarly, if we employ (1.4) with $\mu=0,-1,-2, \ldots$, then, for $\mid p t<T$, we have

$$
\left|e^{\gamma \sqrt{t}}(p t)^{1-\mu / 2} K_{\mu}(2 \sqrt{p t})\right| \leq Q_{\mu}
$$

where $Q_{\mu}$ is independent of $p$ and $t$. 
For $t$ large we employ $(1.7)$ to obtain

$$
\left|e^{\gamma \sqrt{t}}(p t)^{1-\mu / 2} K_{\mu}(2 \sqrt{p t})\right| \leq L_{\mu}|p t|^{-\mu / 2+3 / 4} e^{(\gamma-\operatorname{Re} 2 \sqrt{p}) \sqrt{t}}
$$

where $I_{\mu}$ is again a constant independent of $p$ ana $t$. Let $E_{\mu}=\max \left\{R_{\mu}, Q_{\mu}, L_{\mu}\right\}$. Then

$$
\begin{gathered}
\sup _{0<t<\infty}\left|e^{\gamma \sqrt{t}}(p t)^{1-\mu / 2} K_{\mu}(2 \sqrt{p t})\right| \leq E_{\mu}\left(1+|p t|^{-\mu / 2+3 / 4} e^{(\gamma-\operatorname{Re} 2 \sqrt{p}) \sqrt{t}}\right) \\
\leq E_{\mu}\left(1+|p|^{-\mu / 2+3 / 4}\right)\left(1+|t|^{-\mu / 2+3 / 4} e^{(\gamma-\operatorname{Re} 2 \sqrt{p}) \sqrt{t}}\right)
\end{gathered}
$$

and since $\operatorname{Re} 2 \sqrt{\mathrm{p}}>\gamma$ and $\mu \varepsilon(-\infty, 1)$, it follows that the right hand side of the above expression is less than $c_{\mu}\left(1+|p|^{-\mu / 2+3 / 4}\right)$ with $\mathrm{C}_{\mu}$ a constant depending upon $\mu$. This completes the proof.

We will now show that $\bar{M}_{\mu} f$ is analytic. Namely,

Theorem 3.2. Let $f \in H_{\mu, \gamma}$ and $(\bar{M} f)(p)$ is the Meijer transform of $f$ and $p \varepsilon \Omega_{f}$. Then $\left(\bar{M}_{\mu} f\right)(p)$ is analytic in $\Omega_{f}$ and

$$
D_{p}\left(\vec{M}_{\mu} f\right)(p)=\frac{2}{\Gamma(1-\mu)}<f(t), D_{p^{2}}(p t)^{\mu / 2} K_{\mu}(2 \sqrt{p t})>\text {. }
$$

Proof. For $p \varepsilon \Omega_{f}$, let $c$ be the circle about $p$ of radius $r$ so chosen that it lies entirely in $\Omega_{f}$ and let $|\Delta p|<r_{1}<$ for some $r_{1}$

$\frac{\left(\bar{M}_{\mu} f\right)(p+\Delta p)-\left(\bar{M}_{\mu} f\right)(p)}{\Delta p}-\frac{2}{\Gamma(I-\mu)}\left\langle f(t), D_{p} p(p t)^{\mu / 2} K_{\mu}(2 \sqrt{p t})\right\rangle$

$$
=\left\langle f(t), \phi_{\Delta p}(t)\right\rangle,
$$

where $\phi_{\Delta p}(t)=\frac{2}{\Gamma(1-\mu)}\left[\frac{(p+\Delta p)[(p+\Delta p) t]^{\frac{\mu}{2}} K_{\mu}\left(2 \sqrt{(p+\Delta p) t}-p(p t)^{\frac{\mu}{2}} K_{\mu}(2 \sqrt{p t})\right.}{\Delta p}\right.$

$$
\left.-D_{p} p(p t)^{\frac{\mu}{2}} K_{\mu}(2 \sqrt{p t})\right]
$$

In order to prove the result, it suffices to show that $\phi_{\Delta p}(t) \rightarrow 0$ as $\Delta p \rightarrow 0$ in $M_{\mu, \gamma^{*}}$ Since by Lemma $2.2 D_{p} p(p t)^{\mu / 2} K_{\mu}(2 \sqrt{p t}) \varepsilon M_{\mu, \gamma^{\prime}}$ it follows that (3.4) is well-defined. Further, since for $k=0,1,2, \ldots$ 


$$
B_{-\mu}^{k} D_{p} p(p t)^{\mu / 2} K_{\mu}(2 \sqrt{p t})=D_{p} p^{k+1}(p t)^{\mu / 2} K_{\mu}(2 \sqrt{p t})
$$

by equation (1.5), it follows that $B_{-\mu}^{k} \phi_{1 p}$ is an analytic function of $p$ on $\Omega_{f}$. By Cauchy Integral formula, we have

$$
\begin{aligned}
\mathrm{B}_{-\mu}^{\mathrm{k}} \phi_{\Delta \mathrm{p}}(t)= & \frac{1}{\pi i \Gamma(1-\mu)} \int_{\mathrm{C}} \xi^{\mathrm{k}+1}(\xi t)^{\mu / 2} \mathrm{~K}_{\mu}(2 \sqrt{\xi t}) \times \\
& {\left[\frac{1}{\Delta \mathrm{p}}\left(\frac{1}{\xi-\mathrm{p}-\Delta \mathrm{p}}-\frac{1}{\xi-\mathrm{p}}\right)-\frac{1}{(\xi-\mathrm{p})^{2}}\right] \mathrm{d} \xi } \\
= & \left.\frac{\Delta \mathrm{p}}{\pi i \Gamma(1-\mu)} \int_{C} \frac{\xi^{k+1}(\xi t)^{\mu / 2} \mathrm{~K}_{\mu}(2 \sqrt{\xi t})}{(\xi-p)^{2}(\xi-p-\Delta p)} \mathrm{d}\right) .
\end{aligned}
$$

Since $|\xi-p|=r,|\xi-p-\Delta p|>r-r_{1}$ and $e^{\gamma \sqrt{t}}(\xi t)^{1-\mu / 2} k_{\mu}(2 \sqrt{\xi t})$ is bounded by $c_{\mu}\left(1+|\xi|^{-\mu / 2+3 / 4}\right.$ ) it follows that

$$
\begin{aligned}
\left|e^{\gamma \sqrt{t}} t^{1-\mu_{B} k}{ }_{-\mu} \phi_{\Delta p}(t)\right| & \leq \frac{\left.c_{\mu}\right|_{\Delta p} \mid}{\pi \Gamma(1-\mu)} \int_{C} \frac{\left(1+|\xi|^{-\frac{\mu}{2}+\frac{3}{4}}\left(|\xi|^{k+\mu}\right)\right.}{|\xi-p|^{2}|\xi-p-\Delta p|}|d \xi| \\
& \leq \frac{\left.C_{\mu}\right|_{\Delta p} \mid}{\Gamma(1-\mu)} \frac{2}{r\left(r-r_{1}\right)} \sup _{\xi \varepsilon C}\left(|\xi|^{k+\mu}+|\xi|^{k+\mu / 2+3 / 4}\right)
\end{aligned}
$$

which converges to zero as $\Delta p \rightarrow 0$ and this completes the proof.

The rest of this section is to prove a generalization of the inversion theorem for the classical Meijer transform (3.2) due to Conlan and Koh [1]. Our result is restricted to generalized functions belonging to the space $D(I)$ (Remark 2.2). In particular, we shall prove the following inversion theorem

Theorem 3.3. Let $\left(\bar{M}_{\mu} f\right)(p)$ be the Meijer transform of $f_{\mu, \gamma}$ for $p \varepsilon \Omega_{f}$. Then

$f(t)=\lim _{\theta_{1} \rightarrow \pi} \frac{\Gamma(1-\mu)}{2 \pi i} \int_{-\theta_{1}}^{\theta}\left(\bar{M}_{\mu} f\right)(p) p^{-1}(p t)^{-\mu / 2} I_{\mu}(2 \sqrt{p t}) d p(\theta)$

where $p(\theta)=\frac{\gamma^{2}}{4} e^{i \theta} \sec ^{2} \frac{\theta}{2}$ and $\gamma$ is a fixed real number in $\Omega_{f}$. (The limit is to be understood in the sense of convergence in $\left.D^{\prime}(I)\right)$. 
The proof of Theorem 3.3 will be developed in a sequence of lemmas. We will state the lemmas and give them their proofs in the Appendix.

Lemma 3.2. Let $\phi \varepsilon D(I)$ and $f \varepsilon M_{\mu, \gamma^{*}}$ Then, for a fixed, $\theta_{1} \varepsilon(0, \pi)$,

$$
\begin{aligned}
& \int_{-\theta_{1}}^{\theta} A(p) p<f(t),(p t)^{\mu / 2} K_{\mu}(2 \sqrt{p t})>d p(\theta) \\
& =\left\langle f(t), \int_{-\theta}^{\theta} \mathrm{A}(\mathrm{p}) \mathrm{p}(\mathrm{pt})^{\mu / 2} \mathrm{~K}_{\mu}(2 \sqrt{\mathrm{pt}}) \mathrm{dp}(\theta)\right\rangle
\end{aligned}
$$

where

$$
\begin{aligned}
& A(p)=\int_{0}^{\infty} \phi(t)(p t)^{-\mu / 2} p^{-1} I_{\mu}(2 \sqrt{p t}) d t, \\
& \gamma>\max \left(0, \sigma_{f}\right) \text { and } \operatorname{Re} 2 \sqrt{p}>0 .
\end{aligned}
$$

Lemma 3.3. Let $\phi \varepsilon D(I)$ be such that supp $\phi \subset[A, B]$ and $\sigma$ be a real number such that $0<\sigma<A$. Then for any $\gamma>0$ such that $\operatorname{Re} 2 \sqrt{\mathrm{p}}=\gamma, \quad$ set

$$
Q_{2, \theta_{1}}(\tau)=\int_{(\sqrt{\tau}-\sigma)^{2}}^{(\sqrt{\tau}+\sigma)^{2}} L_{\theta_{1}}(t, \tau) B_{-\mu}^{k} \phi(t) d t,
$$

where

$$
\begin{aligned}
L_{\theta_{1}}(t, \tau)=\frac{1}{\pi i}\left[\frac{t^{-\mu / 2} \tau / 2}{t-\tau}\right. & \sqrt{p}\left(\sqrt{t} I_{\mu+1}(2 \sqrt{p t}) k_{\mu}(2 \sqrt{p \tau})\right. \\
& \left.\left.+\sqrt{\tau} I_{\mu}(2 \sqrt{p t}) k_{\mu+1}(2 \sqrt{p \tau})\right)\right]\left.\right|_{p\left(-\theta_{1}\right)} ^{p\left(\theta_{1}\right)}
\end{aligned}
$$

Then $W_{\theta_{1}}(\tau)=e^{\gamma \sqrt{\tau}} \tau^{1-\mu}\left[Q_{2, \theta_{1}}(\tau)-B_{-\mu}^{k} \phi(\tau)\right]$ converges uniformly to zero on $0<\tau<\infty$ as $\theta_{1} \rightarrow \pi$.

Lemma 3.4. Under the same hypotheses of Lemma 3.3 let

$$
Q_{1, \theta_{1}}(\tau)=\int_{0}^{(\sqrt{\tau}-\sigma)^{2}} I_{\theta_{1}}(t, \tau) B_{-\mu}^{k} \phi(t) d t .
$$

Then $S_{\theta_{1}}(\tau)=e^{\gamma \sqrt{\tau}} \tau^{1-\mu_{Q_{1}}}{ }_{1}(\tau)$ converges uniformly to zero on $0<\tau<\infty$ as $\theta_{1} \rightarrow \pi$.

Lemma 3.5. Under the same hypothese of Lemma 3.3 , let 


$$
\mathcal{Q}_{3, \theta_{1}}(\tau)=\int_{(\sqrt{\tau}+\sigma)^{2}}^{\infty} \mathrm{L}_{\theta_{1}}(t, \tau) B_{-\mu}^{k} \phi(t) d t .
$$

Then $R_{\theta_{I}}(\tau)=e^{\gamma \sqrt{\tau}} \tau^{1-\mu} Q_{3, \theta_{1}}(\tau)$ converges uniformly to zero on $0<\tau<\infty$ as $\theta_{1} \rightarrow \pi$.

Finally,

Lemma 3.6. Let $\phi(t) \varepsilon D(I)$. For a fix $\gamma=\operatorname{Re} 2 \sqrt{p}$, let

$H_{\theta_{1}}(\tau)=\frac{1}{\pi i} \int_{-\theta_{1}}^{\theta}(\mathrm{F} \tau)^{\mu / 2} \mathrm{~K}_{\mu}(2 \sqrt{\mathrm{p} \tau}) \int_{0}^{\infty} \phi(t)(p t)^{-\mu / 2} I_{\mu}(2 \sqrt{p t}) d t d p(\theta)$.

Then for any positive real number $\gamma$ $H_{\partial_{1}}(\tau)$ converges in $M_{\mu, \gamma}$ to $\phi(\tau)$ as $e_{1} \rightarrow \pi$.

Proof of Theorem 3.3. Let $\phi(t) \in D(I)$. We show that

$$
\left.\left\langle\frac{\Gamma(1-\mu)}{2 \pi i} \int_{-\theta}^{\theta} 1 \text { ( } \bar{M}_{\mu} f\right)(p) p^{-1}(p t)^{-\mu / 2} I_{\mu}(2 \sqrt{p t}) d p(\theta), \phi(t)\right\rangle
$$

converges to $\langle f(t), \phi(t)\rangle$ as $\theta_{1} \rightarrow \pi$. Since $(p t)^{-\mu / 2} I_{\mu}(2 \sqrt{p t})$ is a smooth function on $I$, the integral in (3.5) is locally integrable with respect to $t$, it follows that (3.5) can be written as

$$
\begin{aligned}
& \frac{\Gamma(1-\mu)}{2 \pi i} \int_{0}^{\infty} \phi(t) \int_{-\theta_{1}}^{\theta}\left(\bar{M}_{\mu} f\right)(p) p^{-1}(p t)^{-\mu / 2} I_{\mu}(2 \sqrt{p t}) d p(\theta) d t \\
& =\frac{\Gamma(1-\mu)}{2 \pi i} \int_{-\theta}^{\theta} 1 \int_{0}^{\infty} \phi(t) p^{-1}(p t)^{-\mu / 2} I_{\mu}(2 \sqrt{p t}) \cdot \frac{2 p}{\Gamma(1-\mu)}\left\langle f(\tau),(p \tau)^{\mu / 2} z_{\mu}(2 \sqrt{p \tau})\right\rangle
\end{aligned}
$$

$$
\operatorname{dtdp}(\theta)
$$

by Fubini's Theorem and (3.1). For any real number $\gamma$ such that $\gamma>\max \left(0, \sigma_{f}\right)$ Lemma (3.2) implies that $(3.6)$ is equal to $\left.\left\langle f(\tau), \frac{1}{\pi i} \int_{-\theta}^{\theta} 1 \text { (p }\right)^{\mu / 2} K_{\mu}(2 \sqrt{p \tau}) \int_{0}^{\infty} \phi(t)(p t)^{-\mu / 2} I_{\mu}(2 \sqrt{p t}) d t d p(\theta)\right\rangle$ which converges uniformly to $\langle f(\tau), \phi(\tau)\rangle$ in $\mu_{\mu, \gamma}$ as $\theta_{1} \rightarrow \pi$ by Lemma 3.6. Equation (3.5) will then imply that 
$f(t)=\lim _{\theta_{1} \rightarrow \pi} \frac{\Gamma(1-\mu)}{2 \pi i} \int_{-\theta_{1}}^{\theta}(\bar{M} \underset{\mu}{f})(p) p^{-1}(p t)^{-\mu / 2} I_{\mu}(2 \sqrt{p t}) d p(\theta)$

which completes the proof of the theorem.

Remark 3.1. If $\left(\bar{M}_{\mu} f\right)(p)$ and $\left(\bar{M}_{\mu} g\right)(p)$ are the Meijer transforms of $f$ and $g$ for $p \varepsilon \Omega_{f}$ and $p \varepsilon \Omega_{g}$ respectively and if $\left(\bar{M}_{\mu} f\right)(p)=\left(\bar{M}_{\mu} g\right)(p)$ for $p \varepsilon \Omega_{f} \cap \Omega_{g}$, then $f=g$ in the sense of equality in $D^{\prime}(I)$. This uniqueness property is a consequence of Theorem 3.3 .

APPENDIX. In this appendix we shall give the proofs of Lemma 3.2 through Lemma 3.6 .

Proof of Lemma 3.2. Set

$$
N(\tau)=\int_{-\theta_{1}}^{\theta_{1}} A(p) p(p \tau)^{\mu / 2} K_{\mu}(2 \sqrt{p \tau}) d p(\theta) .
$$

Clearly $N(\tau) \in M_{\mu, \gamma^{\circ}} \quad$ This follows from Lemma 3.1

$\lambda \lambda_{\gamma, k}^{\mu}(\mathrm{N}(\tau)) \leq c_{\mu} \int_{-\theta_{1}}^{\theta}|A(p)||p|^{k+\mu}\left(1+|p|^{-\mu / 2+3 / 4}\right)|d p(\theta)|<\infty$.

Let $R(t, m)$ be the Riemann sums for $N(\tau)$ given by

$\left.R(\tau, m)=\frac{e_{1}}{m} \sum_{j=-m}^{m-1} A\left(p\left(\frac{j \theta_{1}}{m}\right)\right) p\left(\frac{j \theta_{1}}{m}\right)\left(\tau p\left(\frac{j \theta}{m}\right)\right)^{\mu / 2} K_{\mu}\left(2 \sqrt{\tau p\left(\frac{j \theta_{m}}{m}\right.}\right)\right)$.

We need to show that for $f \varepsilon M_{\mu, \gamma^{\prime}}<f(t), R(\tau, m)>$ converges uniformly to $\langle f(t), N(\tau)\rangle$. Equivalently, since $f \varepsilon^{\text {in' }} \mu, \gamma$, we need to show that $R(\tau, m)$ converges in $M_{\mu, \gamma}$ to $N(\tau)$ as $m \rightarrow \infty$. That is,

$$
G(\tau, m)=e^{\gamma \sqrt{\tau}} \tau^{1-\mu_{B} k}(N(\tau)-R(\tau, m))
$$

converges uniformly and absolutely to zero on $0<\tau<\infty$ as $m \rightarrow \infty$. Since $\lambda_{r, k}^{\mu}(N(\tau))<\infty$, it follows that for a given $\varepsilon>0$ one can choose a real number $T$ such that for $\tau>T$

$$
\sup _{\tau>T}\left|e^{\gamma \sqrt{\tau}} \tau \tau^{1-\mu_{B} k} N(\tau)\right|<\varepsilon / 2
$$

and for all $\mathrm{m}$ 


$$
\begin{gathered}
\sup _{\tau>T}\left|e^{\gamma \sqrt{\tau}} \tau^{1-\mu_{B} k} R(\tau, m)\right|<\varepsilon / 2\left(\int_{-\theta}^{\theta} 1|A(p)| d p(\theta)\right)^{-1} \frac{\theta}{m} \times \\
\sum_{j=-m}^{m-1}\left|A\left(p\left(\frac{j \theta}{m}\right)\right)\right|
\end{gathered}
$$

which implies that there is $m_{0}$ such that for all $\mathrm{m}>\mathrm{m}_{0}$ (A3) is less than $\varepsilon / 2$. Thus from (A2) and (A3), we have $\sup _{\tau>T} G(\tau, m)<\varepsilon$, for $m>m_{0}$. Now for $0<\tau<T$ and by employing the series expansion of $\mathrm{ik}_{\mu}(2 \sqrt{\mathrm{p} \tau})$ as $\tau \rightarrow 0, \mathrm{e}^{\gamma \sqrt{\tau}} \mathrm{p}^{\mathrm{k}}(\mathrm{p} \tau)^{1-\mu / 2} \mathrm{~K}_{\mu}(2 \sqrt{\mathrm{p} \tau})$ converges uniformly to zero on $\mathrm{p}\left(-\theta_{1}\right)<\mathrm{p}(\theta)<\mathrm{p}\left(\theta_{1}\right)$. If we redefine this function to be zero at $\tau=0$ for the given $p(\theta)$, then $e^{\gamma \sqrt{\tau}} p^{k}(p \tau)^{1-\mu / 2} K_{\mu}(2 \sqrt{p \tau})$ becomes uniformly continuous on $(\tau, p)$ with $0 \leq \tau \leq T$ and $p\left(-\theta_{1}\right)<p(\theta) \leq p\left(\theta_{1}\right)$. Thus $R(\tau, m)$ will converge to the integral and hence there exists $m_{1}$ such that for all $m>m_{1}$, $\sup _{0<\tau<T}(G(\tau, m))<\varepsilon$.

Therefore, for all $\mathrm{m}>\max \left(\mathrm{m}_{0}, \mathrm{~m}_{1}\right), \sup _{0<\tau<\infty}|\mathrm{G}(\tau, \mathrm{m})|<\varepsilon$. That is, $G(\tau, m)$ converges uniformly to zero on $0<\tau<\infty$ as $m \rightarrow \infty$.

In the next three lemmas we shall investigate the boundedness of the integral

$$
\int_{0}^{\infty} I_{\theta_{1}}(t, \tau) B_{-\mu}^{k} \phi(t) d t
$$

where $L_{\theta_{1}}(t, \tau)$ is as defined in Lemma 3.3. Since the proofs of Lemmas $3.3,3.4$ and 3.5 are essentially similar, we shall only give the detailed proof of Lemma 3.3. The idea of the proofs is to use the asymptotic expansions of $K_{\mu}(2 \sqrt{p t})$ and $I_{\mu}(2 \sqrt{p t})$ and get appropriate bounds on the resulting terms.

Proof of Lemma 3.3. Since supp $\phi(t) \subset[A, B]$, it follows that $Q_{2}, \theta_{1}(\tau)=0$ if either $(\sqrt{\tau}+\sigma)^{2} \leq A$ or $(\sqrt{\tau}-\sigma)^{2} \geq B$. Thus we consider $(\sqrt{\mathrm{A}}-\sigma)^{2} \leq \tau \leq(\sqrt{\mathrm{B}}+\sigma)^{2}$. Further, since $p\left(\theta_{1}\right)=\frac{\gamma^{2}}{4} e^{i \theta_{1}} \sec ^{2} \frac{\bar{\theta}_{1}}{2}$, it follows that

$$
|2 \sqrt{\mathrm{pt}}| \geq\left|\gamma \sec \frac{\theta_{1}}{2}\right| \sqrt{\mathrm{A}} \rightarrow \infty, \text { as } \theta_{1} \rightarrow \pi
$$

and

$$
|2 \sqrt{\mathrm{p} \tau}| \geq\left|\gamma \sec \frac{\theta 1}{2}\right||\sqrt{\mathrm{A}}-\sigma| \rightarrow \infty, \text { as } \theta_{1} \rightarrow \pi \text {. }
$$


Employing the asymptotic expansions of $\mathrm{K}_{\mu}(2 \sqrt{\mathrm{pt}})(\operatorname{see}(1.7))$ and of $I_{\mu}(2 \sqrt{p t})$ (see $\left.(1.8)\right)$, we have

$$
\mathrm{w}_{\theta_{1}}(\tau)=\mathrm{J}_{1}+\mathrm{J}_{2}+\mathrm{J}_{3}+\mathrm{J}_{4}
$$

where

$$
\begin{aligned}
& J_{1}=\frac{1}{2 \pi} e^{\gamma \sqrt{\tau}} \tau^{\frac{3}{4}-\frac{\mu}{2}} \int_{(\sqrt{\tau}-\sigma)^{2}}^{(\sqrt{\tau}+\sigma)^{2}} \frac{t^{-\frac{\mu}{2}-\frac{1}{4}} e^{\gamma(\sqrt{t}-\sqrt{\tau})} \sin \left(\gamma(\sqrt{t}-\sqrt{\tau}) \tan \frac{\theta}{2}\right)}{\sqrt{t}-\sqrt{\tau}} \times \\
& \times B_{-\mu}^{k} \phi(t) d t \\
& J_{2}=\frac{1}{2 \pi} e^{\gamma \sqrt{\tau}} \tau^{3 / 4-\mu / 2} \int_{(\sqrt{\tau}-\sigma)^{2}}^{(\sqrt{\tau}+\sigma)^{2}} \frac{t^{-\mu / 2-1 / 4} e^{\gamma(\sqrt{t}-\sqrt{\tau})} \sin \gamma\left((\sqrt{t}-\sqrt{\tau}) \tan \frac{\theta}{2}\right)}{\sqrt{t}-\sqrt{\tau}} x \\
& B_{-\mu}^{k} \phi(t)\left(0\left(|p t|^{-\frac{1}{2}}\right)+0\left(|p \tau|^{-\frac{1}{2}}\right)+0(|p t|)^{-\frac{1}{2}} 0(|p \tau|)^{-\frac{1}{2}}\right) d t
\end{aligned}
$$

and

$$
\begin{aligned}
J_{3}= & -\frac{1}{2 \pi} e^{\gamma \sqrt{\tau}} \tau^{3 / 4-\mu / 2}\left[1+0\left(|p \tau|^{-1 / 2}\right)\right] \\
& \int_{(\sqrt{\tau}-\sigma)^{2}}^{(\sqrt{\tau}+\sigma)^{2} t^{-\mu / 2-1 / 4} e^{-\gamma(\sqrt{t}+\sqrt{\tau})} \cos \left(\gamma(\sqrt{t}+\sqrt{\tau}) \tan \frac{1}{2}-\mu \pi\right.} \\
& \cdot B_{-\mu}^{k} \phi(t)\left[1+0\left(|p t|^{-1 / 2}\right)\right] d t \\
J_{4}= & -e^{\gamma \sqrt{\tau}} \tau^{1-\mu_{B}^{k} k_{-\mu} \phi(\tau) .}
\end{aligned}
$$

We show now that (A4) converges uniformly to zero on $0<\tau<\infty$. Adding $J_{1}$ and $J_{4}$, replacing $x=\sqrt{t}-\sqrt{\tau}$ and adding and subtracting the term

$$
\frac{1}{\pi} e^{\gamma \sqrt{\tau}} \tau^{1-\mu_{B} k_{-\mu} \phi(\tau)} \int_{-\sigma}^{\sigma} \frac{\sin \left(\gamma x \tan \frac{\theta}{2}\right)}{x} d x
$$

we obtain

$$
\begin{aligned}
& \frac{1}{\pi} \int_{-\sigma}^{\sigma} v(x, t) \sin \left(\gamma x \tan \frac{\theta}{2}\right) d x \\
& +e^{\gamma \sqrt{\tau}} \tau^{1-\mu_{B} k} \phi(\tau)\left[\frac{1}{\pi} \int_{-\sigma}^{\sigma} \frac{\sin \left(\gamma x \tan \frac{\theta}{2}\right)}{x} d x-1\right]
\end{aligned}
$$


where

$v(x, \tau)=e^{\gamma \sqrt{\tau}} \tau^{1-\mu} \frac{e^{\gamma x}\left(\frac{x}{\sqrt{\tau}}+1\right)^{-\mu+1 / 2} B_{-\mu}^{k}\left(\phi(x+\sqrt{\tau})^{2}\right)-B_{-\mu}^{k} \phi(\tau)}{x}$

$\mathrm{v}(\mathrm{x}, \tau)$ has a removable singularity at $\mathrm{x}=0$ and is bounded on the domain $\left\{(x, \tau) \mid-\frac{\sqrt{A}}{2}<x<\frac{\sqrt{A}}{2}, \frac{A}{4}<\tau<\left(\sqrt{B}+\frac{\sqrt{A}}{2}\right)^{2}\right\}$. So given any $\varepsilon>0$ we can make the first term in (A5) less than $\varepsilon / 3$ by choosing $\sigma$ small enough, say $\sigma=\sigma_{1}$. The second term of (A5) converges to zero as $\theta_{1} \rightarrow \pi$. Thus $\mathrm{J}_{1}+\mathrm{J}_{4}<\varepsilon / 3$.

In $\mathrm{J}_{2}$ the terms represented by $0\left(|\mathrm{pt}|^{-1 / 2}\right)$ and $0\left(|\mathrm{p} \tau|^{-1 / 2}\right)$ are bounded by $C /\left|1+i \tan \frac{\theta}{2}\right|$ for $C$ sufficiently large. This implies that

$$
\left|\frac{\sin \left(\gamma(\sqrt{t}-\sqrt{\tau}) \tan \frac{\theta}{2}\right)}{\sqrt{t}-\sqrt{\tau}} 0\left(|p t|^{-1 / 2}\right)\right|<C^{\prime}
$$

and

$$
\left|\frac{\sin \left(\gamma(\sqrt{t}-\sqrt{\tau}) \tan \frac{\theta}{2}\right)}{\sqrt{t}-\sqrt{\tau}} 0\left(\left|(p \tau)^{-1 / 2}\right|\right)\right|<c "
$$

where $C^{\prime}$ and $C^{\prime \prime}$ are constants independent of $\theta_{1}$. Therefore the integrand of $\mathrm{J}_{2}$ is bounded on the domain

$$
\left\{(t, \tau) \mid A<t<B ; \quad(\sqrt{A}-\sigma)^{2}<\tau<(\sqrt{B}+\sigma)^{2}\right\} .
$$

This implies that given any $\varepsilon>0$, we can choose $\sigma$ sufficiently small, say $\sigma=\sigma_{2}$ such that $\left|J_{2}\right|<\varepsilon / 3$.

By a similar argument, for $\varepsilon>0$ we can choose $\sigma$ sufficiently small, say $\sigma=\sigma_{3}$. such that $\left|J_{3}\right|<\varepsilon / 3$.

Let $\sigma=\min \left(\sigma_{1}, \sigma_{2}, \sigma_{3}\right)$. Then for a given $\varepsilon>0$ there exists a $\delta>0$ such that $w_{\theta_{1}}(\tau)<\varepsilon$ whenever $\left|\theta_{1}-\pi\right|<\delta$. Equivalently, $\mathrm{w}_{\theta_{1}}(\tau)$ converges to zero on $0<\tau<\infty$ as $\theta_{1} \rightarrow \pi$.

Finally, we shall prove lemma 3.6.

Proof of Lemma 3.6. Since $\phi(t)$ has a compact support and since the integrand is smooth, it follows that

$B_{-\mu}^{k} H_{\theta_{I}}(\tau)=\frac{1}{\pi i} \int_{-\theta_{1}}^{\theta} B_{-\mu}^{k}(p \tau)^{\mu / 2} K_{\mu}(2 \sqrt{p \tau}) \int_{0}^{\infty} \phi(t)(p t)^{-\mu / 2} I_{\mu}(2 \sqrt{p t}) d t d p(\theta)$. 
Using (1.5) and (1.6), we obtain

$B_{-\mu}^{k} H_{\theta_{1}}(\tau)=\frac{1}{\pi i} \int_{-\theta_{1}}^{\theta}(p \tau)^{\mu / 2} K_{\mu}(2 \sqrt{p \tau}) \int_{0}^{\infty} \phi(t)(p t)^{-\mu} B_{-\mu}^{k}(p t)^{\mu / 2} I_{\mu}(2 \sqrt{p t}) d t d p(\hat{\epsilon}$

Integrating the inner integral by parts $2 k$ times, we get

$B_{-\mu}^{k} H_{\theta_{I}}(\tau)=\frac{1}{\pi i} \int_{-\theta_{I}}^{\theta}(p \tau)^{\mu / 2} K_{\mu}(2 \sqrt{p \tau}) \int_{0}^{\infty}(p t)^{-\mu / 2} I_{\mu}(2 \sqrt{p t}) B_{-\mu}^{k} \phi(t) \operatorname{dtdp}(\theta)$

and upon interchanging the order of integration

$B_{-\mu}^{k} H_{\theta_{1}}(\tau)=\frac{1}{\pi i} \int_{0}^{\infty} \int_{-\theta_{1}}^{\theta}(p \tau)^{\mu / 2} K_{\mu}(2 \sqrt{p \tau})(p t)^{-\mu / 2} I_{\mu}(2 \sqrt{p t}) B_{-\mu}^{k} \phi(t) d p(\theta) d t$.

Now, by observing that

$$
\frac{1}{\pi i} \int_{-\theta}^{\theta} I_{1}(p \tau)^{\mu / 2}(p t)^{-\mu / 2} K_{\mu}(2 \sqrt{p \tau}) I_{\mu}(2 \sqrt{p t}) d p(\theta)=L_{\theta}(t, \tau)
$$

(see Erdélyi [3], Equation 9 page 90) where $L_{\theta_{1}}(t, \tau)$ is as in Lemma (3.3), we obtain

$$
B_{-\mu}^{k} H_{\theta_{1}}(\tau)=\int_{0}^{\infty} L_{\theta_{1}}(t, \tau) B_{-\mu}^{k} \phi(t) d t
$$

If $\operatorname{supp} \phi(t)$ is contained in $[A, B]$, then, for $\sigma$ a real number such that $0<\sigma<A, B_{-\mu}^{k}{ }_{\theta_{1}}(\tau)$ can be written as

$$
\begin{aligned}
{ }^{B_{-\mu}{ }^{\mathrm{H}}{ }_{\theta_{1}}(\tau)} & =\int_{0}^{(\sqrt{\tau}-\sigma)^{2}} \cdots d t+\int_{(\sqrt{\tau}-\sigma)^{2}}^{(\sqrt{\tau}+\sigma)^{2}} d t+\int_{(\sqrt{\tau}+\dot{\sigma})^{2}}^{\infty} d t \\
& =Q_{1, \theta_{1}}(\tau)+Q_{2, \theta_{1}}(\tau)+Q_{3, \theta_{1}}(\tau)
\end{aligned}
$$

where $Q_{i, \theta_{1}}(\tau) \quad i=1,2,3$ are as in Lemmas $3.4,3.3$ and 3.5

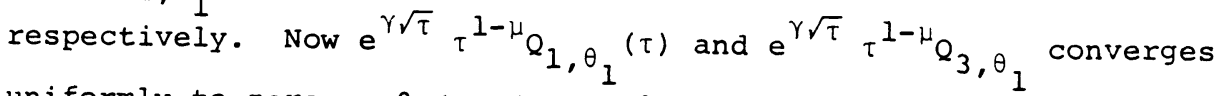
uniformly to zero on $0<\tau<\infty$ as $\theta_{1} \rightarrow \pi$ by Lemma 3.4 and Lemma 3.5 respectively. Moreover, $e^{\gamma \sqrt{\tau}} \tau^{1-\mu}\left[Q_{2, \theta_{1}}(\tau)-B_{-\mu}^{k} \phi(\tau)\right]$ 
converges uniformly to zero on $0<\tau<\infty$ as $\theta_{1} \rightarrow \pi$. Hence

$H_{\theta_{1}}(\tau)$ converges in $H_{\mu, \gamma}$ to $\phi(\tau)$ as $\theta_{1} \rightarrow \pi$. This completes the proof of Lemma 3.6 .

ACKNOWLEDGEMENT. The work of the first author was partially supported by NSERC of Canada under Grant A-7184; that of the second author by

a grant from the University of Houston-Downtown.

\section{REFERENCES} [1] Koh, E.L., A Mikusinski calculus for the Bessel operator $B_{\mu}$, Proc. D1ff.
Eq., Springer-Verlag Lect. Notes $\$ 564(1976), 291-300$.

[2] Conlan, J. and E.L. Koh, On the Meijer transformation, Internat. J. Math. and Math. Sci., Vol. 1 (1978), 145-159. [3] Zemanian, A.H., Generalized Integral Transformations, Inter-Science,
New York, 1968.

[4] Dube, L.S. and J.N. Pandey, On the Hankel transform of distributions, Tohoku Math. J., 27 (1975), 337-354.

[5] McBride, A.C., The Hankel transform of some classes of generalized functions and connections with fracticnal inipgration. Proc. Roy.
Soc. Edinburgh. 81A (1978), 95-117.

[6] Koh, E.L., The n-dimensional distribuitional Hankel transformacion, Can. J. Math., 27(2) (1975), 423-433.

[7] Watson, G.N., A Treatise on the Theory of Bessel Functions, University Press, Cambridge, 1966.

[8] Erdélyi, A, et al, Higher Transcendental Functions, Vol. II, MCGraw Hill, New York, 195,3. 


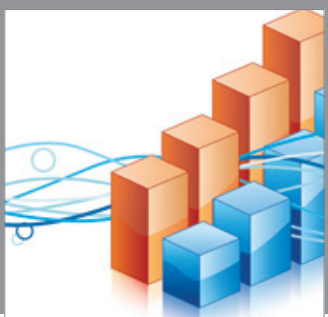

Advances in

Operations Research

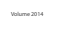

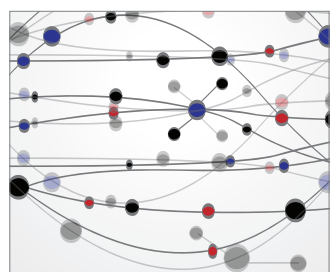

\section{The Scientific} World Journal
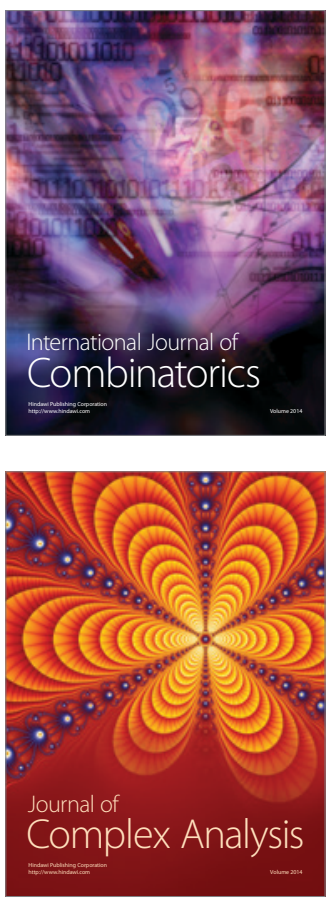

International Journal of

Mathematics and

Mathematical

Sciences
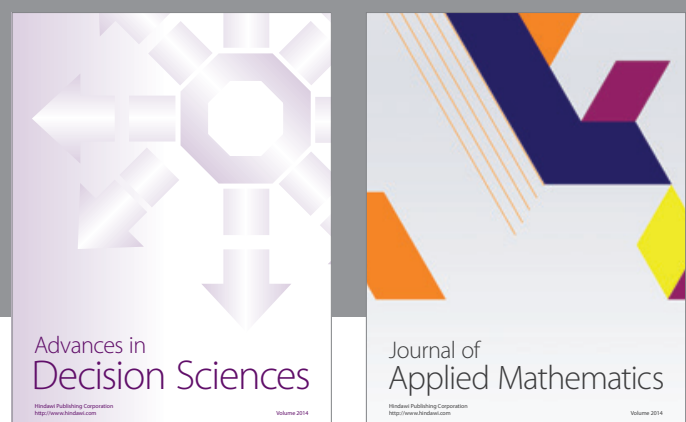

Journal of

Applied Mathematics
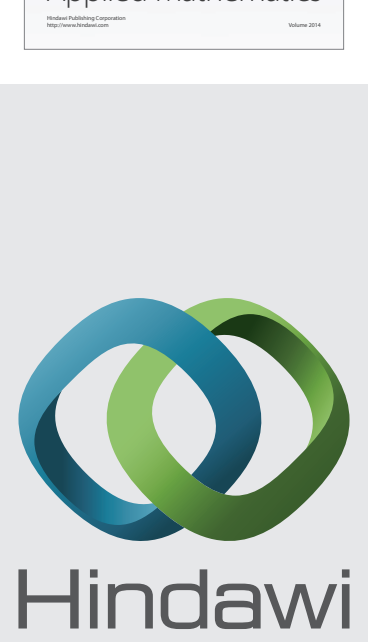

Submit your manuscripts at http://www.hindawi.com
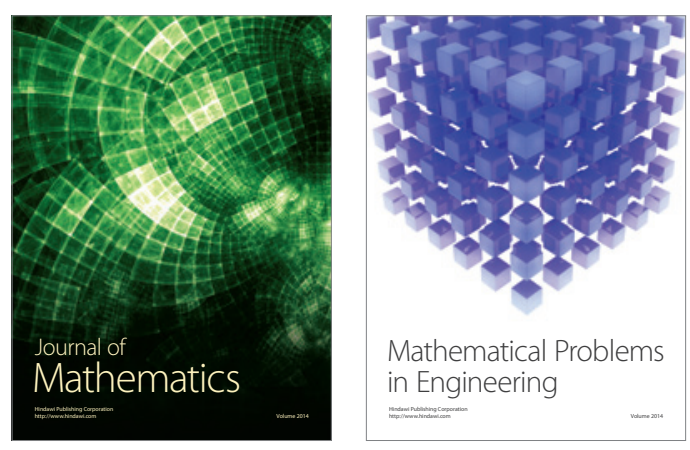

Mathematical Problems in Engineering
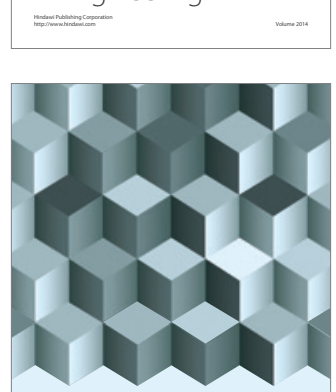

Journal of

Function Spaces
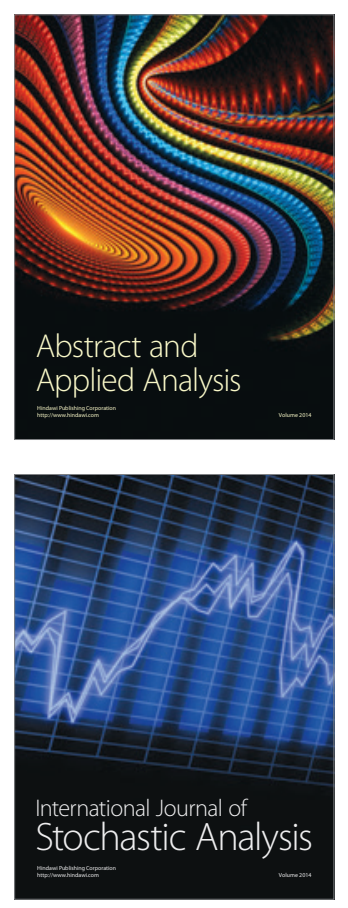

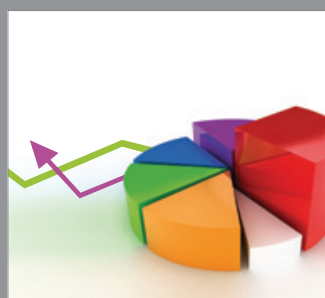

ournal of

Probability and Statistics

Promensencen
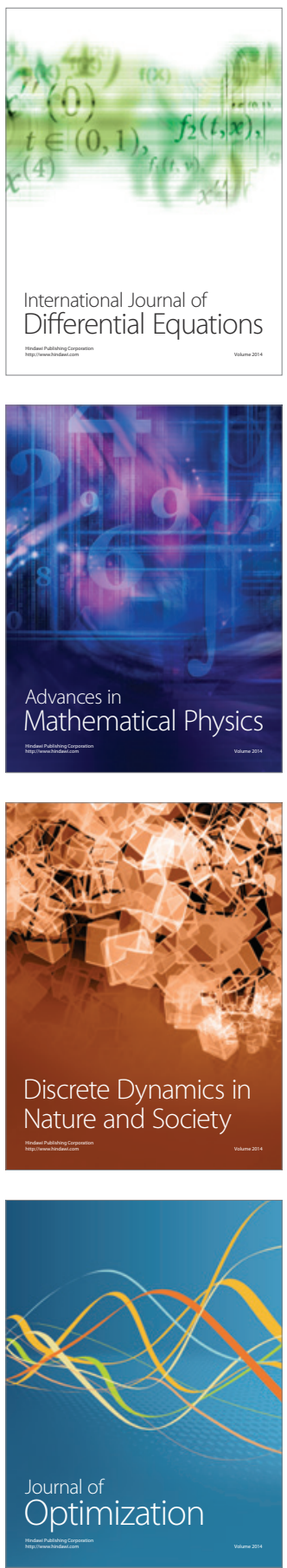